\title{
Trial-by-trial dynamics: a window in time
}

\author{
Jesse J. Bengson ${ }^{1}$ and Ali Mazaheri ${ }^{2 *}$ \\ Center for Mind and Brain, University of California at Davis, Davis, CA, USA \\ 2 Donders Institute for Brain, Cognition and Behaviour, Radboud University, Nijmegen, Netherlands \\ ${ }^{*}$ Correspondence: ali.mazah@gmail.com
}

\section{A commentary on:}

Maladaptation of event-related EEG responses preceding performance errors by Heike Eichele, Hilde T. Juvodden, Markus Ullsperger and Tom Eichele, Front. Hum. Neurosci. (2008) 4:12. doi: 10.3389/ fnhum.2010.00065.

The arrow of time is of little consequence to the majority of physics. Except in a few cases, the laws of physics still apply whether one observes them in a reverse or in a forward manner. The contrasting notion relevant to this discussion is that our experience of time flows uni-directionally into the future. If our experimental approaches manifest as a function of our experience, then it has been an unsurprising technique in Cognitive Neuroscience to observe the consequences of an act of cognition in the direction of time's arrow: i.e., to observe the neural correlates of cognition after an event of interest. With few exceptions (Remington, 1969; Squires et al., 1976) this influential approach has characterized most of cognitive neuroscience. The recent paper by Eichele et al. (2010) represents a methodological reversal of time's arrow to illustrate the conditions that precede a cognitive event of interest. The authors' examine how brain activity under the control of a previously established performance monitoring network can serve to define an error-prone brain state well in advance of an imminent error. This approach offers insight into the dynamic and adaptive properties of the brain that would otherwise be relegated to the status of unexplained variance and gives an avenue for possible insight into causal, rather than associated mechanisms of perception and action.

A classic approach within human electrophysiology has been to link the neural consequences of cognition to an averaged event-related signal that differs as a function of an a priori experimental manipulation. The most conventional approach here has been to use averaged event-related responses to characterize the brain's transient response to the event. However, the neuronal activity prior to the onset of the event exhibits large fluctuations on time scales of hundreds of milliseconds to seconds. As such, a burgeoning direction of research has examined how this pre-stimulus activity influences perception, cognition, and behavior. The underlying hypothesis here is that the fluctuations in pre-stimulus neural activity reflect brain states that influence the moment-to-moment processing, as well as later cognitive and motor decisions and outputs. The majority of the studies in this fledgling field (Eichele et al., 2010, being a notable exception) have largely focused on the spontaneous variability of neuronal fluctuations indexed by oscillatory activity. Several recent studies point to visual and somato-sensory perception being modulated by pre-stimulus oscillatory activity. In particular, activity in the alpha band $(\sim 10 \mathrm{~Hz})$ has been shown to predict failures in visual (Ergenoglu et al., 2004; Thut et al., 2006; van Dijk et al., 2008) and somato-sensory perception (Linkenkaer-Hansen et al., 2004). Moreover, fluctuations in alpha activity have also been found to predict failures in sustained attention (Mazaheri et al., 2009; O'Connell et al., 2009). The work of Eichele represents an exceptional extension of this approach into a clever examination of the pre-trial behavioral and stimulus contingencies as related to the evolution of the evoked response and the subsequent commission of an error. This approach manifests primarily through the re-operationalization of an experimental condition as a function of stimulus contingencies and subsequent behavior rather than an a priori-experimental manipulation. If our goal is to model neural activity at the systems level as a function of cognition and behavior, a combination of the classic approach with this trial by trial dynamic approach would prove a powerful avenue by which to pursue the stubborn question of how the brain, perception, and behavior interact.

An interesting question for future research arises as a consequence of Eicheles' and others' work concerning trial by trial dynamics of perception and behavior relates directly to the work of Squires referenced in the quote at the beginning of Eichele et al.:

\begin{abstract}
"It is hardly surprising to find that the organism's response to "identical" stimuli is in flux. The nervous system is not a passive recipient of inputs that are obediently switched to outputs; rather it is a dynamic system that continuously generates hypotheses about the environment" (Squires et al., 1976)
\end{abstract}

A fundamental question is how the same stimulus (an incompatible trial), and presumed act of cognition (appropriate categorization with reference to task instructions), and identical behavior (a correct response) across a number of seemingly identical trials can be so highly variable in terms of neural activity. Surely, the notion of hypothesis generation in the act of perception is of note here; but a more fundamental aspect of redundancy in the neural systems associated with perception and action must be at play. The question is: how can the cognitive system do a seemingly identical operation repeatedly in a different way each time? The question itself puts strict limits on the computer metaphor of the mind and the answer will illuminate a fundamental property of the mind-brain association.

\section{REFERENCES}

Eichele, H., Juvodden, H., Ullsperger, M., and Eichele, T. (2010).Maladaptation of event-related EEG responses preceding performance errors. Front. Hum. Neurosci. 4, 12. doi: 10.3389/fnhum.2010.00065.

Ergenoglu, T., Demiralp, T., Bayraktaroglu, Z., Ergen, M., Beydagi, H., and Uresin, Y. (2004). Alpha rhythm of the EEG modulates visual detection performance in humans. Brain Res. Cogn. Brain Res. 20, 376-383. 
Linkenkaer-Hansen, K., Nikulin, V.V., Palva, S., Ilmoniemi, R. J., and Palva, J. M. (2004). Prestimulus oscillations enhance psychophysical performance in humans. J. Neurosci. 24, 10186-10190.

Mazaheri, A., Nieuwenhuis, I. L., van Dijk, H., and Jensen, O. (2009). Prestimulus alpha and mu activity predicts failure to inhibit motor responses. Hum. Brain Mapp. 30, 1791-1800.

O’Connell, R. G., Dockree, P. M., Robertson, I. H., Bellgrove, M. A., Foxe, J. J., and Kelly, S. P. (2009). Uncovering the neural signature of lapsing attention: electrophysiological signals predict errors up to 20 s before they occur. J. Neurosci. 29, 8604-8611.
Remington, R. J. (1969). Analysis of sequential effects in choice reaction times. J. Exp. Psychol. 82, 250-257.

Squires, K. C., Wickens, C., Squires, N. K., and Donchin, E. (1976). The effect of stimulus sequence on the waveform of the cortical event-related potential. Science 193, 1142-1146.

Thut, G., Nietzel, A., Brandt, S. A., and Pascual-Leone, A (2006) Alpha-band electroencephalographic activity over occipital cortex indexes visuospatial attention bias and predicts visual target detection. J. Neurosci. 26, 9494-9502.

van Dijk, H., Schoffelen, J. M., Oostenveld, R., and Jensen, O. (2008). Prestimulus oscillatory activity in the alpha band predicts visual discrimination ability. J. Neurosci. 28, 1816-1823.

Received: 17 September 2010; accepted: 30 September 2010; published online: 02 November 2010.

Citation: Bengson JJ and Mazaheri A (2010) Trial-by-trial dynamics: a window in time. Front. Hum. Neurosci. 4:199. doi: 10.3389/fnhum.2010.00199

Copyright (c) 2010 Bengson and Mazaheri. This is an open-access article subject to an exclusive license agreement between the authors and the Frontiers Research Foundation, which permits unrestricted use, distribution, and reproduction in any medium, provided the original authors and source are credited. 